\title{
ANEW IMPROVED TACTIC TO EXTRACT FACIAL EXPRESSION BASED ON GENETIC ALGORITHM AND WVDF.
}

\author{
Md Alamgir Hossain ${ }^{1}$, Prof (Dr) Goutam Sanyal ${ }^{2}$ \\ ${ }^{1}$ Department of Computer Application (MCA), CIT, West Bengal, India \\ alamgir_simeyahoo.com \\ ${ }^{2}$ Department of CSE, NIT, Durgapur, West Bengal, India \\ nitgsanyal@gmail.com
}

\begin{abstract}
The study and analysis of facial expression has paying attention tremendously as human being is very conscious in the domain of computer vision because it takes a principal role in the area of man-machine communication. Almost all types of face expressions are basically spawned by taking a new hue of facial muscles, which grades in temporally malformed facial features such as forehead, eye-lids, eye-brows, nose, lips, and skin colour/texture, etc. During the evaluation procedure of any facial expression emphasise should have to be given on the following parameters :( a) the parts of the face that will play prime role in facial action, $(b)$ the intensity of facial actions, and $(c)$ the stochastic parameters of facial actions. In our paper we have proposed a real-time, precise-exact, and robust facial expression recognition methodology depending on genetic algorithm along with Weighted Vector Directional Filters (WVDF) as it is proved that it is the most powerful unbiased optimization techniques for sampling a huge solution space. The GA can be optimized by using WVDF filter's coefficients. By using the Stochastic Selection of parents and the generation of optimal offspring, the optimal WVDF filter can be obtained. We have been capable to construct a leaf-matrix to our required intended expression. Ultimately, we have evaluated our proposed methodology by using Weighted Vector Directional Filters (WVDF) along with genetic algorithm with the shutter function of Canon Camera. We have accomplished our job by achieving better performance than Sony on slight function.
\end{abstract}

\section{KEYWORDS}

WVDF filter, WVDFM, leaf-matrix, genetic algorithm, pattern matching, face-detection.

\section{INTRODUCTION}

In the recent past a considerable amount of research work has been done depending on behaviour of human being. The researchers are devoting their involvement to pull out the facial expression in a variety of ways. There are two main procedural approaches to determine the abovementioned three characteristics of facial expressions: signed based approaches and judgement based approaches. The first approaches are concerned with the facial actions based on the coding method, where the actions of the faces are extracted and explained by their poses, locations and intensities of face-action-coding method. We have proposed in our paper a very promoting realtime, accurate and robust facial expression detection procedure depending on the Weighted Vector Directional Filters (WVDF) along with genetic algorithm accompanied by the shutter function. Detection of different types of actions of human being is the intended mission aiming at the security surveillance work in the domain of digital image processing. The facial expression DOI : 10.5121/ijait.2012.2504 
International Journal of Advanced Information Technology (IJAIT) Vol. 2, No.5, October 2012

identification methodology is executed based on three parameters: recognition of face, features extraction of the face images and face-state categorization and reshuffling. The methodological information for the exposure of face-state is a must at the time when we have the requirement to identify the location of any face within a set of images. The procedure to build them, scale them and render those images for the research work of facial image detection a thorough knowledge should have to be kept. In our proposed methodology we have built a leaf-matrix to categorize all types of expressions. Finally, we have evaluated our proposed methodology by using Weighted Vector Directional Filters (WVDF) accompanied by the genetic algorithm and the shutter function of Canon Camera. However, we have established that a better feat can be obtained in accordance with the shutter function of Canon than Sony on slight.

\section{RELATED WORK}

The problem related to the detection such as happiness, anger, surprise, fear, etc are the road ways by which we can recognize the facial expressions. There are many scholastic researches who have paved their ways on facial expression recognition, but there is not much researchers who have shown their interest in the process of detection of different facial expressions based on some sophisticated algorithms like Genetic Algorithm. Youngkyoon Jang and Woontack Woo [3] proposed an algorithm that can identify a lip feature point in almost all types of lip without depending on colour. Therefore, this algorithm makes a rope to adaptive feature recognition by real-time study based on histogram. Jaakko Astola, Perti Haavisto, and Yrjo Neuvo [5] proposed that if probability density function is applied based on likelihood estimation approach then the achieved result will be nearer to VCM. Rastislav Lukac, Bogdan Smolka, Konstantinos N. Plataniotis, and Anastasios N. Venetsanopoulosa [6] have proposed that WVDFs depending on the collection of the yielded sample of multichannel series of input can be optimized. Based on weighted coefficients of WVDFs accompanied with different parameters a set of smooth operations can be performed. An angular optimization algorithm is designed to take advantage of adaptive stack filters and weighted median filtering framework. The optimized WVDFs is efficient to remove image noise with a diversity of signal and noise information. J. Chanussot, M. Paindavoine and P. Lambert [7] proposed a multicomponent image is obtained after decoding based on filtering technique and this can be applied in case of colour images and they have worked on the real time implementation depending on filtering procedure. Rustisluv Lukuc, Bogdun Smolku, Konstuntinos N. Plutuniotis, Anustusios N. Venetsunopoulos and Puvol Zuvarsky [8] proposed a method to adapt a nonlinear filtering technique based on behavior of stochastic signal and noise statistics. They have shown how to reduce noise from an image by the vector directional filtering methodology. Sony's shutter algorithm and detection rate are not quietly sufficient enough to access the position. Sensing Component Company Omron has lately released smile measurement software. It can automatically detect and identify faces of one or more people and assign each smile a factor from $0 \%$ to $100 \%$. Omron uses 3D face mapping technology and claims that its detection rate is more than $90 \%$. But since it is not accessible and we are not in the position to test how it could be performed. Therefore, we have tested our program with normal camera and have proved that we have a better performance on detecting slight smile and lesser false alarm rate on grimace expressions.

\section{WVDF}

The use of WVDF filter's coefficients in optimized way with the help of different mathematical approaches has given a new hue globally as it can be used in optimal way depending on weighted coefficient vector. Along with genetic algorithm if we can apply Weighted Vector Directional Filtering methodology (WVDFM) then that will be a very new methodology for detection and recognition of different types of facial poses, expressions, etc because it is proved that WVDF is the most authoritative impartial optimization methodology for examining a large clarification 
space as it is used taking coefficients as parameters. If Stochastic Selection of parents are used and the production of optimal offspring are considered based on WVDFM filtering technique then we can obtain optimal result. During the experiment we should have to consider the sets weighted angular distances as a whole taking them as input samples. The weighted coefficients of the WVDFs are considered to achieve a numeral of smooth-operations which can be reused for unambiguous filtering scenarios. The methodology can also be commenced a widespread WVDF structure to filter colour image if they are represented in vectors directional method.

\section{ALGORITHM FOLLOWED}

The modelling of any specific and related problem depending on any precise field with the help of genetic algorithm proves fabulous endeavour along in the human computer vision. Modelling escorts the assortment of free model constraints, their coding methodology and as well as the formulation of a definite functions in an optimized way. An uncomplicated genetic algorithm can be represented accompanied by the following five steps:

1. Firstly, we should have to start with a randomly generated population of $\mathrm{N}$ chromosomes, where $\mathrm{N}$ is total population, $\mathrm{L}$ length of chromosome $\mathrm{C}$.

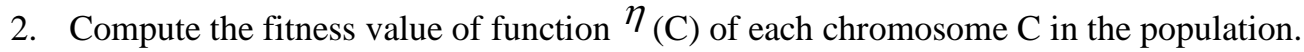

$F(p)=\left\{\begin{array}{ll}0 & p=0 \\ F(p-1)+f(p-1) & 1 \leq p \leq K_{\max }-K_{\min }\end{array}---(1)\right.$

Where $K_{\max }$ and $K_{\min }$ represent maximum and minimum intensity values.

Repeat until N off springs is created:

3. Probabilistically select a pair of chromosomes randomly from population in stock by using value of fitness function.

4. Construct an offspring $\mathrm{Q}_{\mathrm{m}}$ using crossover and mutation operators, where $\mathrm{m}=1,2 \ldots \mathrm{N}$. WVDF filters use a positive real weight coefficient vector $V=\left(V_{1}, V_{2} \ldots V_{N}\right)$ coupled of test images' vectors $\mathrm{C}_{1}, \mathrm{C} 2 \ldots \mathrm{C}_{\mathrm{N}}$. Each weight-vector component consists of one image pixel. Weighted coefficients' vector is analogous to the feature vector considered for testing. The output of filter $\mathrm{Q}=\mathrm{Cm} \in \mathrm{V}$ minimizes the summation of the total angular distances to supplementary test images inside the window $\mathrm{V}$.

$$
\underset{C_{m} \in}{\operatorname{minag}} \sum_{p=1}^{N} \operatorname{VpZ}(\mathrm{Cm}, \mathrm{Cp}) \text { for } \mathrm{m}=1,2 \ldots \mathrm{N} \text {---------- (2) }
$$

Where $\mathrm{Z}(\mathrm{Cm}, \mathrm{Cp})$ represents the angle between the two color vectors $\mathrm{CL}=(\mathrm{Cm} 1, \mathrm{Cm} 2$, $\mathrm{Cm} 3)$ and

5. Replace present population with recently formed one.

6. Go to step 2. 


\section{FLOWCHART OF PROPOSED ALGORITHM}

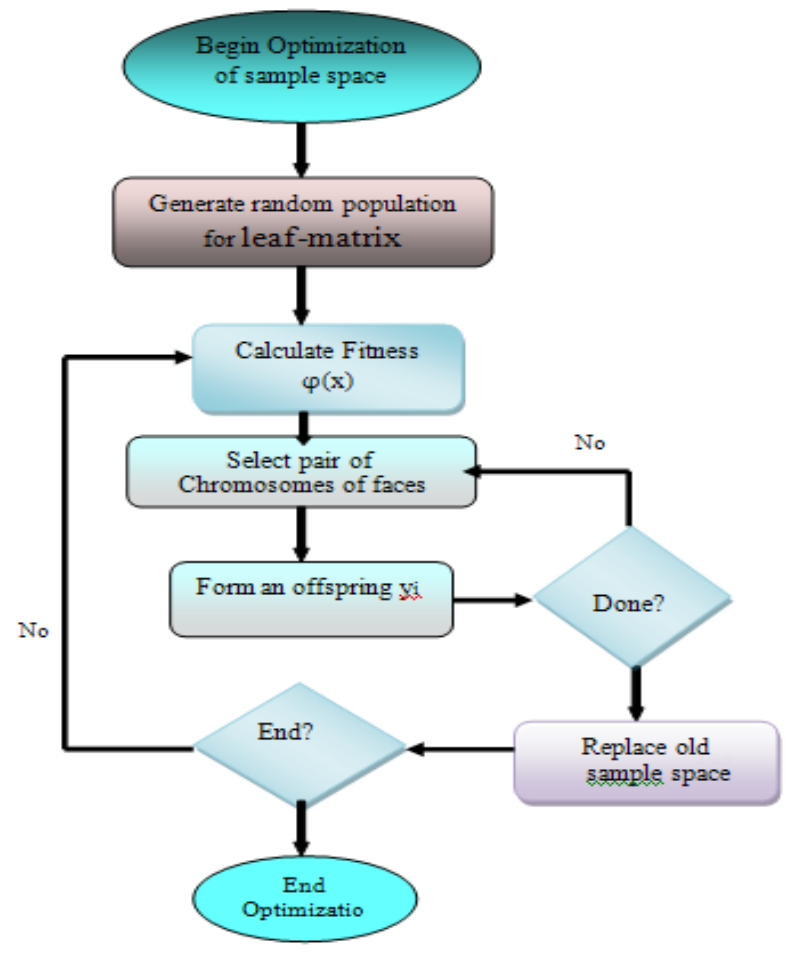

\section{FEATURES EXTRACTION}

During the features extraction procedure we shall have to considered face first and then we have to transfer the image into binary form (as our experiment is basically based on colour image). From those converted-binary images the centroid (Ym, Yn) of the face-image is calculated with the help of the following equations:

$$
\begin{aligned}
& Y_{m}=\frac{\sum P Q}{\sum P} \cdots \cdots(3) \\
& Y_{n}=\frac{\sum P T}{\sum P}-------(4)
\end{aligned}
$$

Where (Ym, Yn) is the co-ordinate value and $\mathrm{P}=\mathrm{G}(\mathrm{G}, \mathrm{T})=0$ or 1.Then from the centroid, entirely the whole face has been cropped and converted into the gray level and the features have been collected.

\section{EXPERIMENTAL RESULT}

In our experiment we have used the Genetic Algorithm along with WVDF methodology to gain better and accurate results with colour images as well as the images that have enriched with noise and have different posed as well as have angular distance. The following Face-images and others also have been used to test our proposed methodology. 
International Journal of Advanced Information Technology (IJAIT) Vol. 2, No.5, October 2012

First we have a get the Normal face (Fig1, Fig 3, Fig 5) and another Smile face (Fig2, Fig4, Fig6). Extraction of a facial expression based of Genetic Algorithm and WVDFM produces the following outputs.

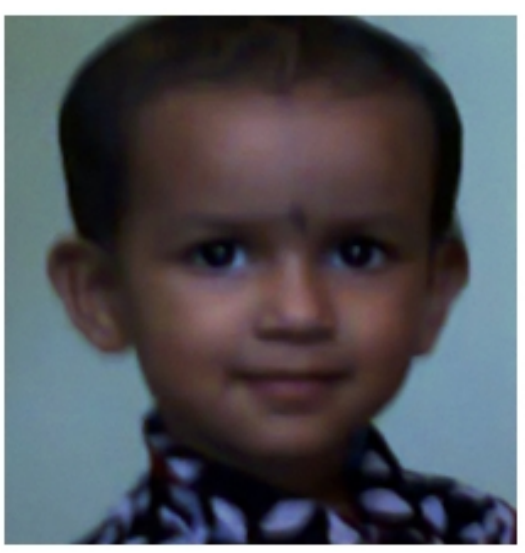

Fig1: Face image without WVDF

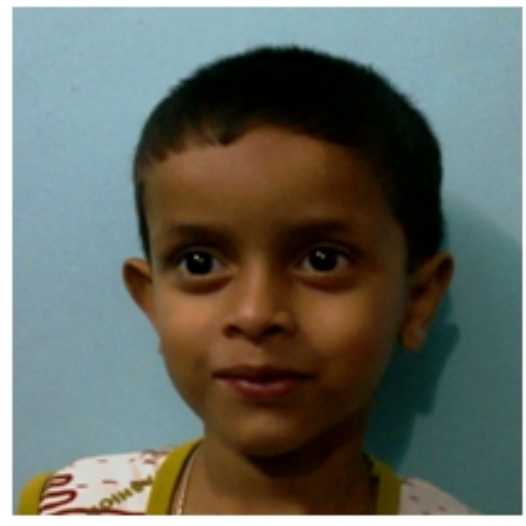

Fig3: Face image without WVDF

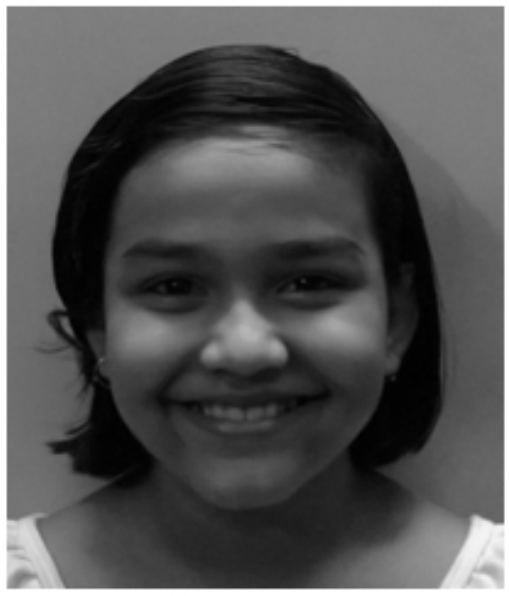

Fig5: Face image without WVDF

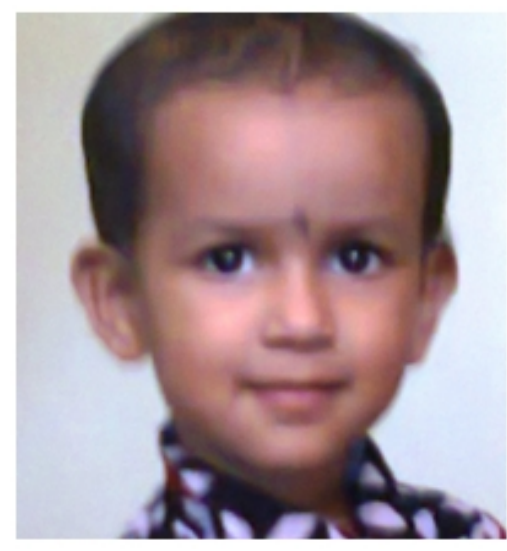

Fig2: Face image with WVDF

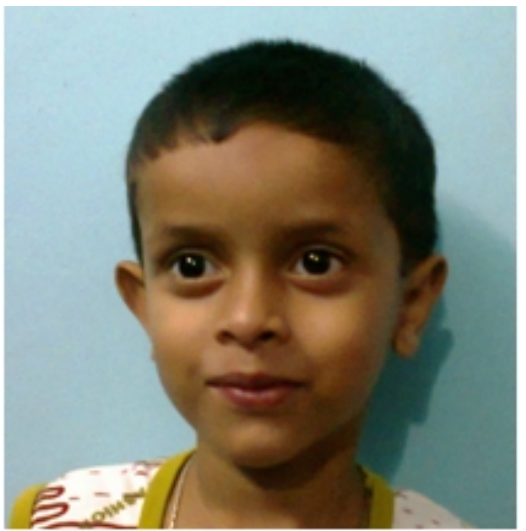

Fig4: Face image with WVDF

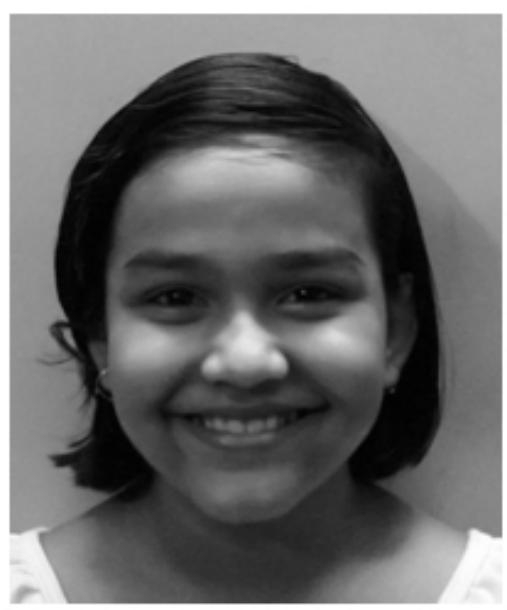

Fig6: Face image with WVDF 
International Journal of Advanced Information Technology (IJAIT) Vol. 2, No.5, October 2012

\begin{tabular}{|c|c|c|c|c|}
\hline $\begin{array}{c}\text { No. of } \\
\text { Face Image }\end{array}$ & $\begin{array}{c}\text { Successfully } \\
\text { Recognized } \\
\text { Face Image }\end{array}$ & $\begin{array}{c}\text { Unrecognized } \\
\text { Face Image }\end{array}$ & $\begin{array}{c}\text { Efficiency in (\%) } \\
\text { by using GA only }\end{array}$ & $\begin{array}{c}\text { Efficiency in (\%) by } \\
\text { using GA+WVDF }\end{array}$ \\
\hline 7 & 5 & 2 & $\mathbf{7 1 . 4 2}$ & 75.31 \\
\hline 21 & 20 & 1 & $\mathbf{9 5 . 2 3}$ & 99.22 \\
\hline 25 & 17 & 8 & $\mathbf{6 8}$ & 71.90 \\
\hline 29 & 29 & 0 & $\mathbf{1 0 0}$ & $\mathbf{1 0 0}$ \\
\hline 34 & 31 & 3 & $\mathbf{9 1 . 1 7}$ & 95.07 \\
\hline 48 & 37 & 11 & $\mathbf{7 7 . 0 8}$ & $\mathbf{8 0 . 9 8}$ \\
\hline
\end{tabular}

Table 1: Results for GA \& WVDF on Face extraction

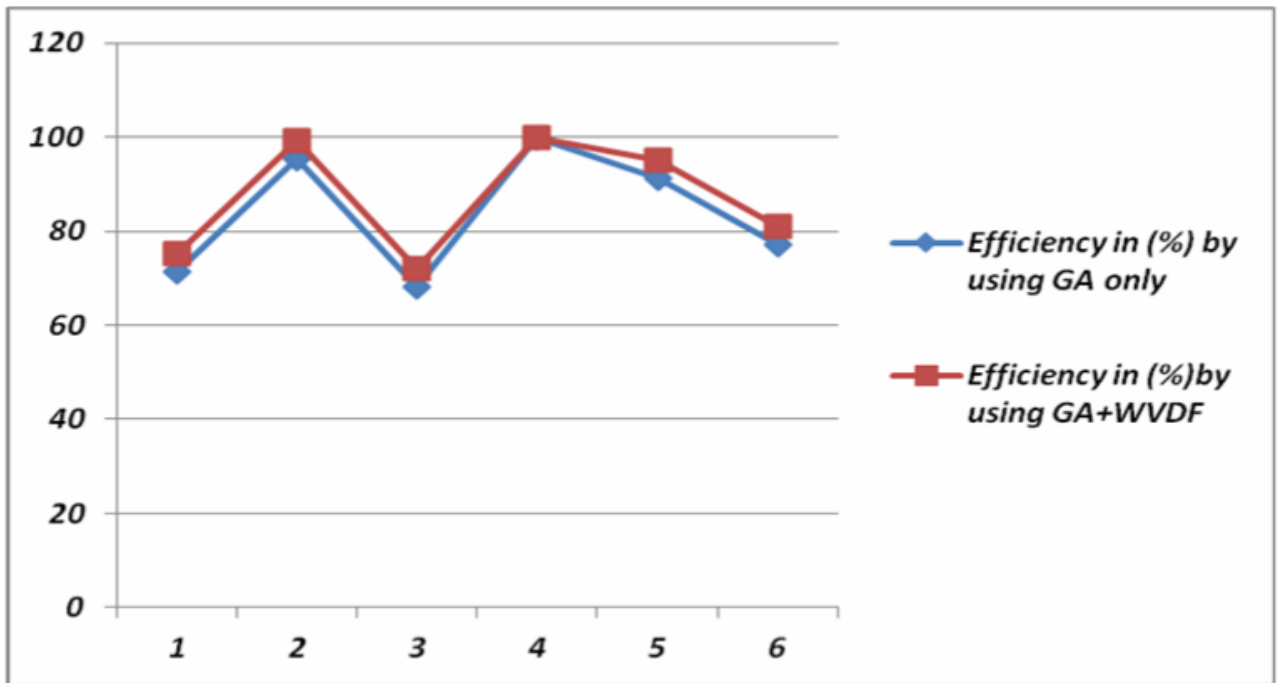

Table 2: Efficiency Chart based on GA and WVDF

\section{CONCLUSION}

In our present thesis paper, a robust and sophisticated methodological technique has been used to Recognize Facial Expression Scheme by using WVDF filtering methodology accompanied by Genetic algorithm. A leaf-matrix has been proposed and digital image processing has been discussed. A stochastic as well as static Facial Expression Recognition technique has been developed herewith. The maximum efficiency is $100 \%$ for Face Recognition System by using Genetic algorithm and the minimum efficiency is $68 \%$. This efficiency rate has been increased by the approximation of $4 \%$ in almost all sample images by using WVDF. Apart from using WVDF there are a lot of parameters involved that could be used along with the sample images that will lead to achieve better results. The efficiency can also be increased by using better scanner, better technique for scaling and efficient technique of edge detection such as advanced edge detection technique and feature extraction of the face image and by using better pre-processing techniques. We shall try to incorporate them in our future work. 
International Journal of Advanced Information Technology (IJAIT) Vol. 2, No.5, October 2012

\section{REFERENCES}

[1] J.T. Alander. Indexed bibliography of genetic algorithms in signal and image processing. University of Vaasa, Department of information technology and production economics, 2003.

[2] Rafael C. Gonzalez and Richard E Woods," Digital Image Processing Using MATLAB7”, Person Education Asia.

[3] Youngkyoon Jang and Woontack Woo, "Adaptive Lip Feature Point Detection Algorithm for RealTime Computer Vision-Based Smile Training System “,M. Chang et al. (Eds.):Edutainment 2009, LNCS 5670, pp. 379-389, 2009. (C) Springer-Verlag Berlin Heidelberg 2009.

[4] S. Rajasekaran \& G.A. Vijayalakshmi Pai, "Neural Networks, Fuzzy Logic and Genetic Algorithms" Prentice-Hall of India Private Limited, 2003.

[5] Jaakko Astola, Perti Haavisto, Yrjo Neuvo," Vector Median Filters", 0018-9219/90/04000678\$01.00@1990 IEEE, PROCEEDINGS OF THE IEEE,VOL.78,NO.4,APRIL 1990.

[6] Rastislav Lukac, Bogdan Smolka,Konstantinos N. Plataniotis, and Anastasios N. Venetsanopoulosa "Selection weighted vector directional filters". 1077-3142/\$ - see front matter _ 2003 Elsevier Inc. All rights reserved. doi:10.1016/j.cviu.2003.10.013Pg- Computer Vision and Image Understanding 94 (2004) 140-167

[7] J. Chanussot M. Paindavoine P. Lambert, " Real Time Vector Median Like Filter FPGA Design and Application to Color Image Filtering”, Pg-0-7803-5467-2/99/ \$10.00 01999 IEEE.

[8] Rustisluv Lukuc, Bogdun Smolku, Konstuntinos N. Plutuniotis ,Anustusios N. Venetsunopoulos and Puvol Zuvarsky , “ANGULAR MULTICHANNEL SIGMA FILTER”,Pg- 0-7803-7663-3/03/\$17.00 02003 IEEE 111 - 745 ICASSP 2003.

[9] Md. Murad Hossain and Md. Shamsul Alam,"BD FINEREADER: A Bangla Character Recognition System for Converting Printed Bangla Text Documents into Editable Electronics Documents", ICCIT-2006.

[10] Y. Wang, H. Ai, B. Wu, and C. Huang, "Real time facial expression recognition with adaboost," in Proceedings of the 17th International Conference on Pattern Recognition (ICPR 2004), 2004.

[11] M. Pantic and J. Rothkrantz, "Facial action recognition for facial expression analysis from static face images," IEEE Transactions on Systems, Man and Cybernetics, vol. 34, no. 3, 2004.

[12] Y. Tian, T. Kanade, and J. Cohn, "Recognizing action units for facial expression analysis," IEEE Transactions on Pattern Analysis and Machine Intelligence, vol. 23, no. 2, 2001.

[13] A. Kapoor, Y. Qi, and R. Picard, "Fully automatic upper facial action recognition," in IEEE International Workshop on Analysis and Modelling of Faces and Gestures, 2003.

[14] I. Kotsia and I. Pitas, "Facial expression recognition in image sequences using geometric deformation features and support vector machines,'IEEE Transactions on Image Processing, vol. 16, no. 1, 2007.

[15] L. G. Shapiro, and G. C. Stockman, Computer Vision,Prentice-Hall Inc., 2001.

[16] R. Chellappa, C.L. Wilson, S. Sirohey (1995), "Human and machines recognition of faces: a survey", Proc. IEEE 83(5): 705-740.

[17] A.Samal and P.A.Iyengar (1992): "Automatic recognition and analysis of human faces and facial expressions: A survey. Pattern Recognition”.

[18] Tian, Y., Kanade, T., Cohn, J.F.: Recognizing Action Units for Facial Expression Analysis. IEEE Transactions on Pattern Analysis and Machine Intelligence 23(2), 97-115 (2001)

[19] Tian, Y., Kanade, T., Cohn, J.F.: Robust Lip Tracking by Combining Shape, Color and Motion. In: ACCV 2000, pp. 1040-1045 (2000).

[20] M. Gudmundsson, E.A. El-Kwae, M.R. Kabuka. Edge detection in medical images using a genetic algorithm. IEEE transactions on medical imaging, 1998, Vol.17, No.3, 469 - 474.

[21] J. Guzaitis, A. Verikas. Image analysis and information fusion based defect detection in particleboards. Electronics and electrical engineering, Kaunas: Technological, 2006, No. 7(71), 67 72.

[22] N.R. Harvey, S. Marshall. The design of different classes of morphological filter using genetic algorithms. IEEE fifth international conference on image processing and its applications, 1995, 227 231. 


\section{AUTHORS PROFILE:}

Md. Alamgir Hossain has received his MCA degree from University of North Bengal and M.Tech degree from Bengal Engineering and Science University, Shibpur (BESUS), West Bengal. He possesses an experience of more than 14 years in the field of teaching and research. He has published more than 9 papers in International Journals and Conferences. He is pursuing his $\mathrm{PhD}$ in Computer Science and Engineering from National Institute of Technology, Durgapur, India in the area of Image Processing. His areas of interest are Artificial Intelligence, Natural Language Processing and Image Processing. He has guided more than 25 PG and $40 \mathrm{UG}$ projects and thesis. He is presently working as an Assistant Professor of Calcutta Institute of Technology under the department of Computer Application (MCA), Uluberia, Howrah, West Bengal, India

Prof (Dr) Gautam Sanyal is a member of the IEEE. He has received his B.E and M.Tech degree from National Institute of Technology (NIT), Durgapur, India. He has received Ph.D.(Egg.) from Jadavpur University, Kolkata, India, in the area of Robot Vision. He possesses an experience of more than 25 years in the field of teaching and research. He has published more than 100 papers in International and National Journals and Conferences. three $\mathrm{PhD}$ (Egg) have already been awarded under his guidance. At present he is guiding another six $\mathrm{PhD}$ scholars in the field of steganography, Cellular Network, High Performance Computing and Computer Vision. He has guided over 10 PG and 130 UG thesis. His research interests include Natural Language Processing, Stochastic modelling of network

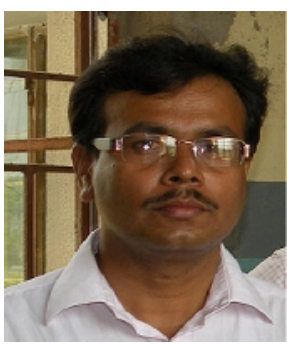
traffic, High Performance Computing, Computer Vision. He is presently working as a Professor in the department of Computer Science and Engineering and also holding the post of Dean (Students' Welfare) at National Institute of Technology, Durgapur, India. 